\title{
Gain Enhancement of Horn Antenna Using Meta Surface Lens
}

\author{
R Manikandan ${ }^{1}$, P K Jawahar ${ }^{2}$ and P H Rao ${ }^{3}$ \\ ${ }^{1}$ Department of Electronics and Communication Engineering \\ B.S. A. Crescent University, Chennai, India, \\ ${ }^{2}$ Department of Electronics and Communication Engineering \\ B.S. A. Crescent University, Chennai, India \\ ${ }^{3}$ Electromagnetics and Antenna Division, SAMEER - Centre for Electromagnetics, Chennai, India \\ Corresponding authors: manikandan 84 @gmail.com, jawahar@bsauniv.ac.in
}

\begin{abstract}
Three dimensional meta surface lens is proposed to improve the gain and beam shaping of horn antenna. Hence an array of SRR as a meta surface lens has been designed, fabricated and investigated. This meta surface lens can be used to convert the spherical wave to plane wave for a wide range of frequency. It is proved by permeability and permittivity of meta surface. In this work the operating bandwidth of the proposed antenna is in the range of $9 \mathrm{GHz}$ to $11 \mathrm{GHz}$ for satellite application. The radiation pattern of $\mathrm{E}$ and $\mathrm{H}$ plane is sharpened in this work. The antenna $3 \mathrm{~dB}$ beam width, and front to back ratio were $9.2^{\circ}, 28 \mathrm{~dB}$ at $10 \mathrm{GHz}$. The radiation characteristics of horn antenna with meta surface have been studied numerically and confirmed experimentally, showing an average gain improvement of $\sim 3 \mathrm{~dB}$ with respect to horn antenna without meta surface lens.
\end{abstract}

Keyword: 3D Meta surface lens, array of SRR, negative permittivity, satellite application.

\section{Introduction}

With the growing need of inexpensive, simple, compact high directional antenna, pyramidal horn antenna may be the best solution. Its robustness and simplicity make pyramidal horn antenna to use widely in microwave and millimeter wave systems, especially used in calibration standard due to its predictability [1].

In the past decade engineers are finding a new technique to enhance the directivity of antenna, particularly for satellite reflector feed. In such case meta surface lens play a key role in reducing side lobe levels in radiation characteristics. Since meta surface lens convert the spherical wave into plane wave in minimum [2 - 6]. This paper presents a new technique for improving the performances of pyramidal horn antenna with meta surface lens.
In recent years the realization of negative refractive index media has been made possible by developments in the field of meta surface. A meta surface is a composite material, as it contains some conductors are arranged in a proper lattice over the dielectrics in order to give rise to dielectric and magnetic properties which is not present in natural material. For wavelength much longer than the lattice spacing the electromagnetic response is averaged over the cells giving rise to value of effective dielectric constant $\varepsilon(\omega)$, magnetic permeability $\mu(\omega)$ and refractive index $n(\omega)$ that are not obtainable in nature [7]. This kind of material can be easily designed and fabricated for particular range of frequency with unusual characteristics, like negative effective permeability and permittivity that make the wave propagation characteristics [8].Pyramidal antenna performance can be improved using the proposed approach of sharpening field at E-plane and H-plane. By keeping the meta surface lens in the near field of horn antenna to suppress the side lobe of the far field radiation pattern [11]. Gain enhancement of millimeter wave horn antenna [12] is achieved with hybrid design, combines the Soret and fishnet metamaterial lens.

In section II, Numerical simulation of unit cell with SRR is presented and reviewed its critical parameter like relative permeability and permittivity. In section III, 2D numerical result for the $\mathrm{X}$ - band horn antenna with meta surface lens are presented and discussed. Experimental results of horn antenna with and without meta surface are presented in section IV.

\section{Unit Cell of Meta surface Design}

The split ring resonator has become common element as that repeated element in meta surface that exhibits magnetic and electric property. A medium in which SRR are periodically positioned can be approximately characterized by the following frequency dependent permeability $\mu(\omega)$ and permittivity $\varepsilon(\omega)$.

To study the characteristic of planar periodic structures in HFSS, Floquet ports are exclusively used. Unit cell can be analyzed in HFSS with Floquet port with specialized 
boundary condition instead of analyzing infinite array periodic structure. Master and slave boundaries enable us to model planes of periodicity where the E-Field on one surface matches the E-field on another to within a phase difference at each corresponding point on the master boundary. In this setup HFSS performs a modal decomposition that gives information of the radiating structure in the form of S-matrix. This S-matrix will interrelate all wave modes and all Floquet modes in the model. Assume that electromagnetic wave propagate through z-axis, the applied magnetic field lies along the SRR (x-axis) and electric field line along the y-axis in the SRR plane. While SRR medium is known to have a predominately magnetic response, it concern with the phase advantage of wave propagation with in the medium. Effective refractive index of the meta surface medium is $(\eta)$, relative impedance is $(\mathrm{z})$, relative permeability $\left(\mu_{\mathrm{r}}\right)$ and relative permittivity $\left(\varepsilon_{\mathrm{r}}\right)$. The value of $\eta, \mathrm{z}, \mu_{\mathrm{r}}$ and $\varepsilon_{\mathrm{r}}$ can be extracted from the $S$-parameter assuming that the unit cell test is symmetric with respect to the $(\mathrm{y}-\mathrm{z})$ plane which means $\mathrm{S}_{11}=\mathrm{S}_{22}$ and $\mathrm{S}_{21}=\mathrm{S}_{12}$. The law of refraction is used for analysis. Deflection of ray at the interference can be calculated with help of low refractive index. The relative permittivity and permeability can be given with respect to the $\mathrm{S}$ - parameter using the following equation and calculated values are shown in figure 1.

In this work numerical simulation is performed with Ansys HFSS 16.0 for proposed horn antenna with and without meta surface lens.

$$
\begin{aligned}
& \mu_{\mathrm{r}}=\frac{2}{\mathrm{jk} \mathrm{k}_{0} \mathrm{D}} \frac{1-\left(\mathrm{s}_{21}-\mathrm{s}_{11}\right)}{1+\left(\mathrm{s}_{21}-\mathrm{s}_{11}\right)} \\
& \varepsilon_{\mathrm{r}}=\frac{2}{\mathrm{j} \mathrm{k}_{0} \mathrm{D}} \frac{1-\left(\mathrm{s}_{21}+\mathrm{s}_{11}\right)}{1+\left(\mathrm{s}_{21}+\mathrm{s}_{11}\right)} \\
& \mathrm{z}= \pm \sqrt{\frac{(1+\mathrm{S} 11)^{2}-\mathrm{S} 21^{2}}{(1-\mathrm{S} 11)^{2}-\mathrm{s} 21^{2}}} \\
& n=\left[\frac{\mathrm{S} 21}{1-\mathrm{S} 11 \frac{\mathrm{z}-1}{\mathrm{z}+1}}\right] \frac{1}{\mathrm{k}_{0} \mathrm{~d}}
\end{aligned}
$$

where,

$\mathrm{k}_{0}$ - free space propagation, $\mathrm{D}$ - is the thickness of the unit cell

The meta surface lens consist of array of split ring resonator on the FR4 with relative permittivity $\varepsilon_{\mathrm{r}}=4.4$ and dielectric loss tangent $\delta=0.02$, the thickness of the substrate is $1.6 \mathrm{~mm}$. The simulated unit cell of SRR on HFSS has shown very good results, a wide pass band is observed in the range of $9 \mathrm{GHz}$ to $11 \mathrm{GHz}$, which can be used to achieve antenna directivity and gain enhancement with narrow beam. Figure 1(a) presents the real and imaginary part of relative permittivity and permeability respectively in the frequency range of $9 \mathrm{GHz}$ to $11 \mathrm{GHz}$. The real and imaginary values of effective index of refraction are shown in figure 1(b). From the figure it is evident that real value is less than land close to zero at the range of frequencies from 9.7 to $10.8 \mathrm{GHz}$. This metamaterial has minimum index of refraction is 0.14 . The characteristics of meta surface lens are studied from dispersion diagram (phase (deg.) with respect to frequency) and plotted in figure 1(c). This dispersion values calculated numerically by following equation,

$$
\beta p=\cos ^{-1}\left(\frac{1-\left(s_{11} s_{22}+s_{12} s_{21}\right)}{2 s_{21}}\right)
$$

The figure 1(c) gives the additional proof for fast wave nature of meta surface lens. From the figure it is observed that, phase advance of 82 degree is achieved with meta surface lens due to low refractive index.

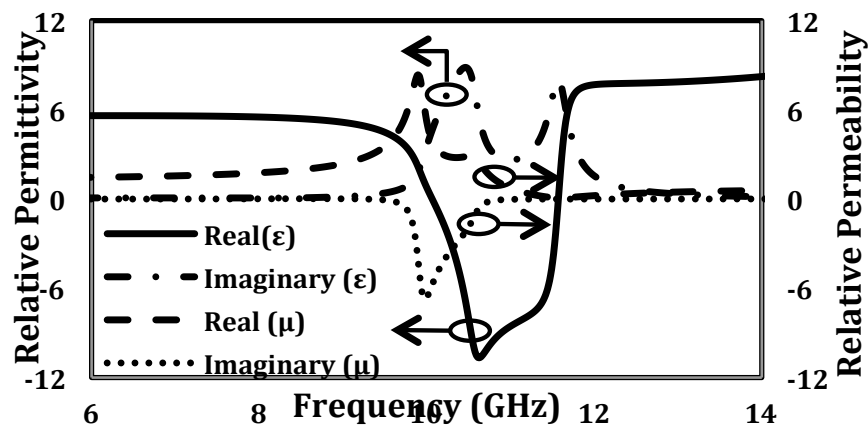

Figure 1(a): Extracted real and imaginary part of relative permittivity and relative permeability of unit cell with respect to frequency.

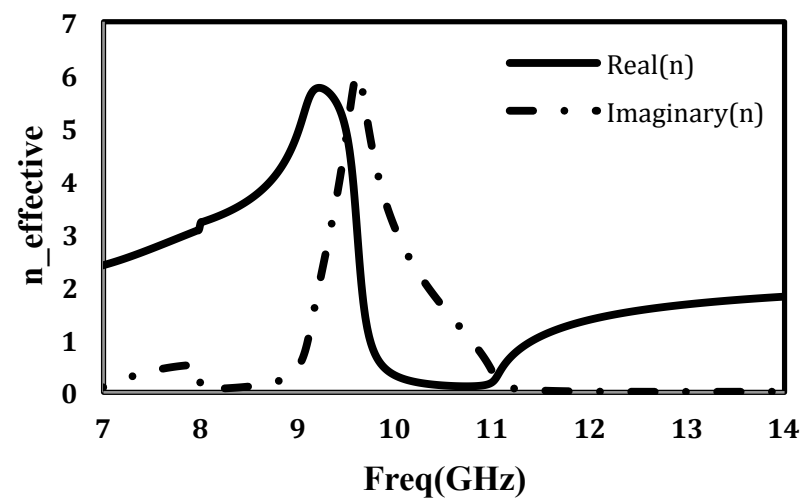

Figure 1(b): Extracted real and imaginary part of effective refractive index of unit cell with respect to frequency.

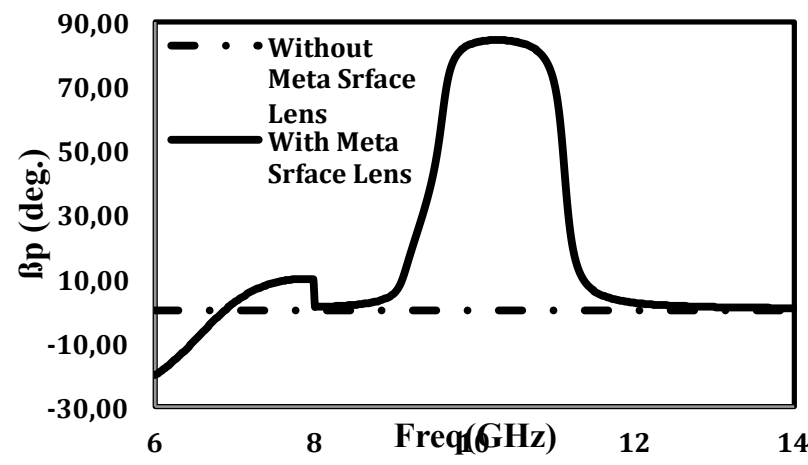

Figure 1(c): Phase variation of electromagnetic wave when passes through the unit cell with respect to frequency. 


\section{Design of Meta Surface lens - Loaded Horn Antenna}

A prototype pyramidal horn antenna is designed based on WR-90 standard for X band frequency range and numerical simulation with HFSS software package have been carried out. Horn antenna is fabricated with aluminum material.

The meta surface lens is kept in the near field of the horn antenna and various analysis has been done with respect to gap(d) between the horn and meta surface lens. Referring to figure 2, the geometric dimensions of the analyzed horn antenna are chosen as L1 $=30 \mathrm{~mm}, \mathrm{~L} 2=285 \mathrm{~mm}, \mathrm{~A}=$ $150 \mathrm{~mm}, \mathrm{~B}=125 \mathrm{~mm}, \mathrm{a}=22.86 \mathrm{~mm}, \mathrm{~b}=10.16 \mathrm{~mm}, \mathrm{t}=3.63$ $\mathrm{mm}, \mathrm{g} 1=0.5 \mathrm{~mm}, \mathrm{~g}_{2}$ and $\mathrm{g}_{3}=10 \mathrm{~mm}$. Meta surface lens consist of array of SRR (unit cell) which is presented in figure 1 with horn antenna. Figure 3 present the $S_{11}$ on the aperture for the normal impedance with and without meta surface lens.

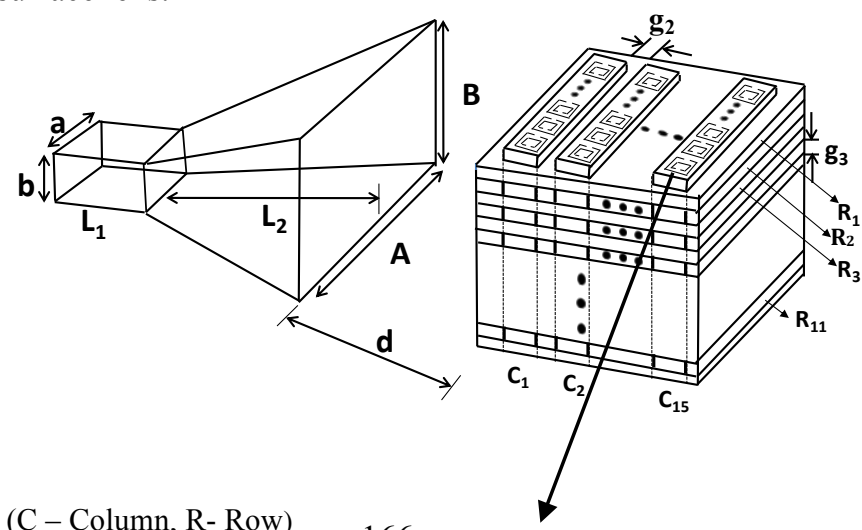

(C - Column, R- Row) 166
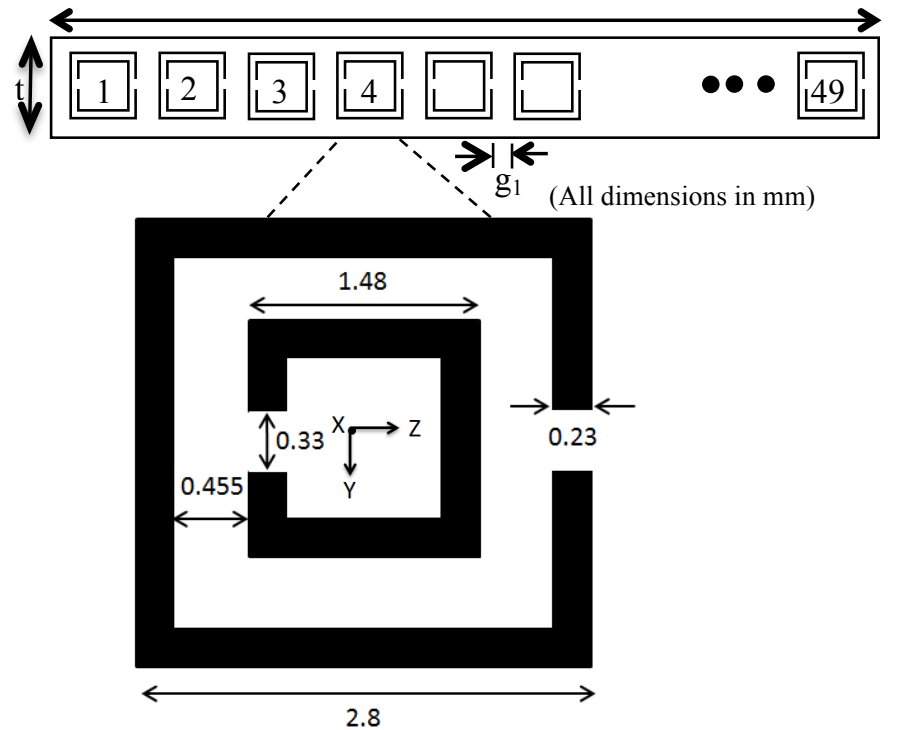

Figure 2: Pyramidal horn antenna and 3D meta surface lens and Unit cell with SRR

In this case $\mathrm{S}_{11}$ lies between -17 and -25 in the operating frequency of $9 \mathrm{GHz}$ to $11 \mathrm{GHz}$. In the proposed approach the aperture fields are manipulated by using 3D meta surface lens and succeeded in improving directivity of horn antenna. Geometry of horn with meta surface lens illustrated in figure
2. The meta surface lens consist of array of SRR on a strip of FR4. Strips are arranged with the gap $g_{2}$ and $g_{3}$ on to achieve the directivity and gap is filled with foam which is having dielectric constant is approximately equivalent to 1 .

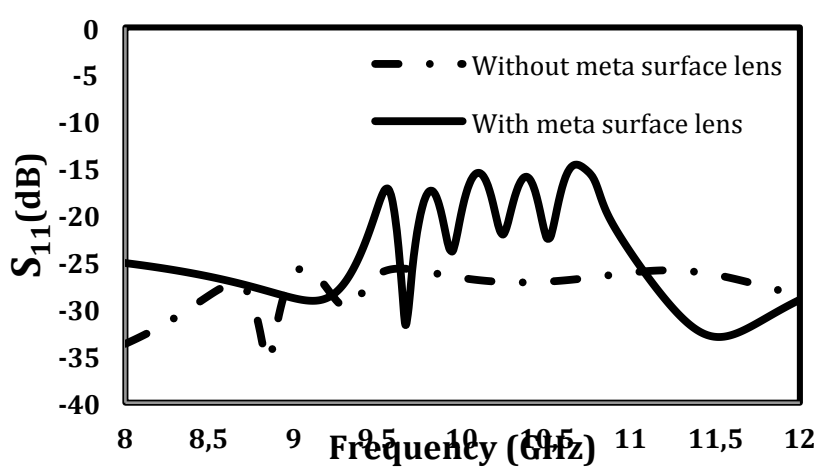

Figure 3: $\mathrm{S}_{11}$ of horn Antenna with and without meta surface lens.

This meta surface lens is arranged in such a way that the amplitude of E field gets tapered with uniform phase distribution to get high directivity with narrow beam width. For the fixed resonance frequency of $10 \mathrm{GHz}$. Figure 4(a), 4(b), 4(c) and 4(d) illustrates the relation between gain and number of meta surface strip arranged in column and row. Gain of horn antenna is $25 \mathrm{~dB}$ and $22 \mathrm{~dB}$ with and without meta surface lens. Gain increases when meta surface lens placed in front of horn antenna.

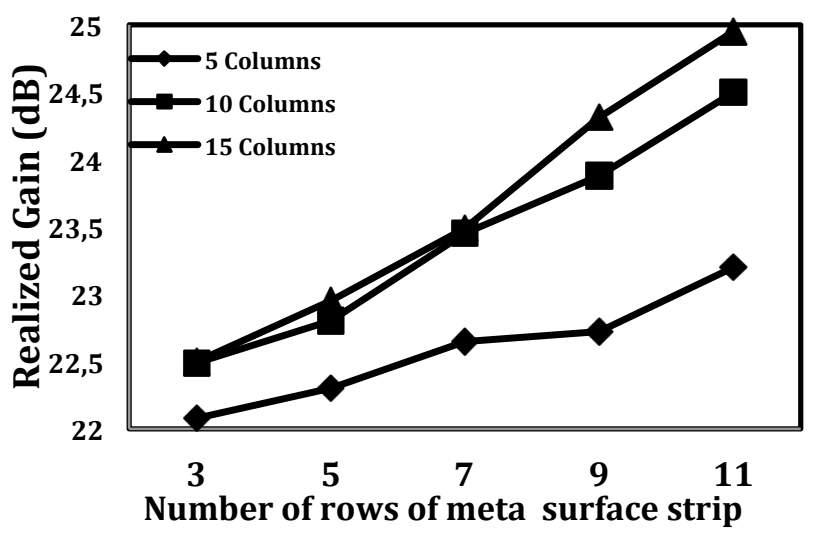

(a)

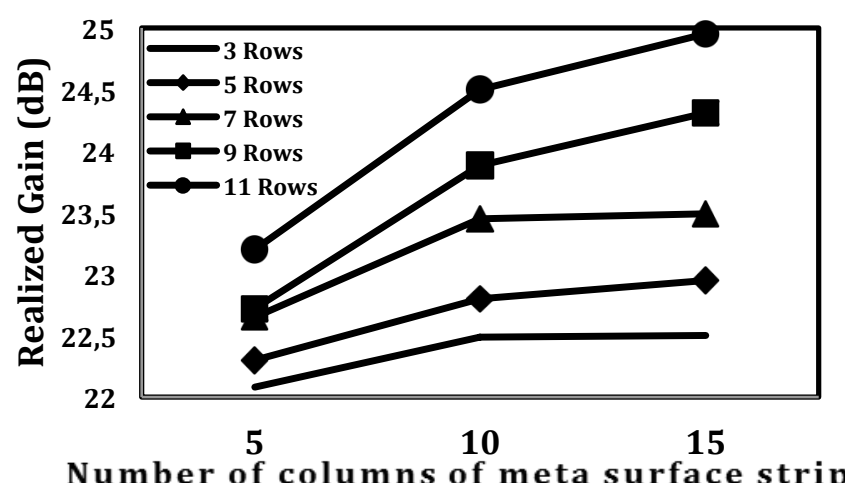

(b) 


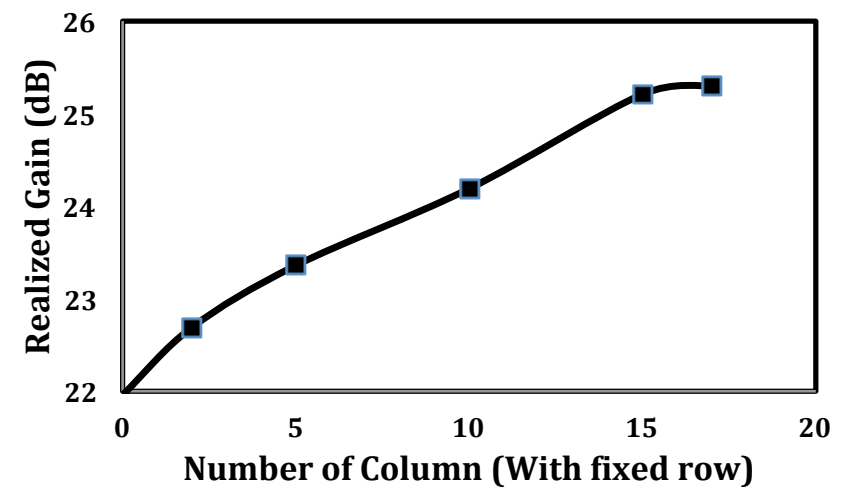

(c)

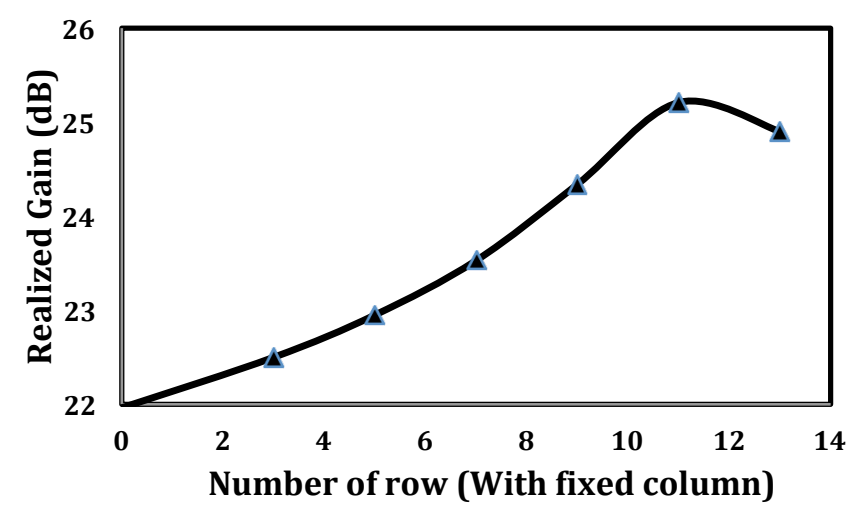

(d)

Figure 4: Simulated realized gain with respect to number of column and row meta surface lens at $10 \mathrm{GHz}$ respectively. (a) Gain versus number of columns of strip, (b) gain versus number of rows of strip, (c) gain versus number of columns of strip with fixed row and (d) gain versus number of rows of strip with fixed column.

Meta surface lens consist of FR4 strip, by increasing the meta surface strip with respect to column gain rise up and get saturated when more than 15 columns are added. But in case of row, gain rise up with respect to number of rows of strip and fall down when aperture of lens increase's beyond the aperture of horn antenna which is presented in figure 4(c) and 5(d). The simulated radiation pattern of the horn antenna with and without meta surface lens at far field presented in figure 5 at the two sample frequencies $(9.7 \mathrm{GHz}$ and $10 \mathrm{GHz}$ ).

It is observed that in the far field, horn antenna (with meta surface lens) beam width in the E- plane is narrower than $\mathrm{H}$ Plane and also gain is enhanced. It has apparently lower side lobe when it is compared with the horn antenna without meta surface lens. Also gain drops down due to non-uniform phase distribution when the meta surface lens aperture is increased higher than the horn aperture which is presented in figure 4.

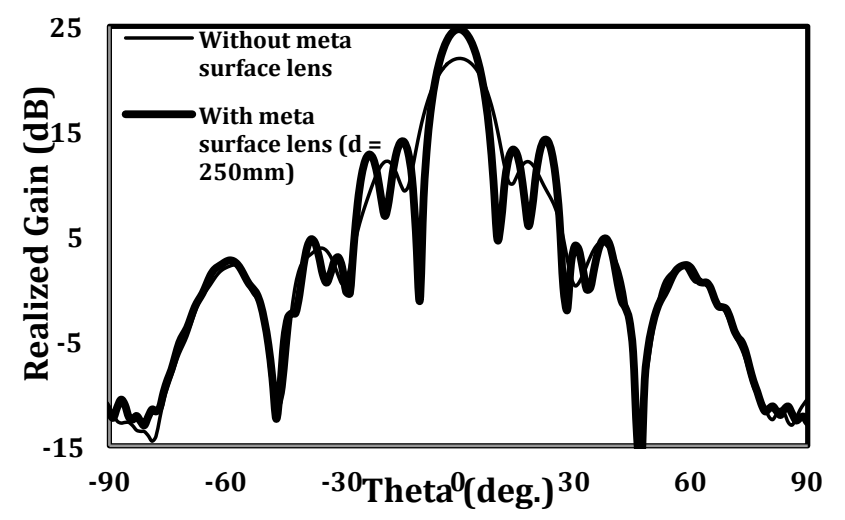

(a)

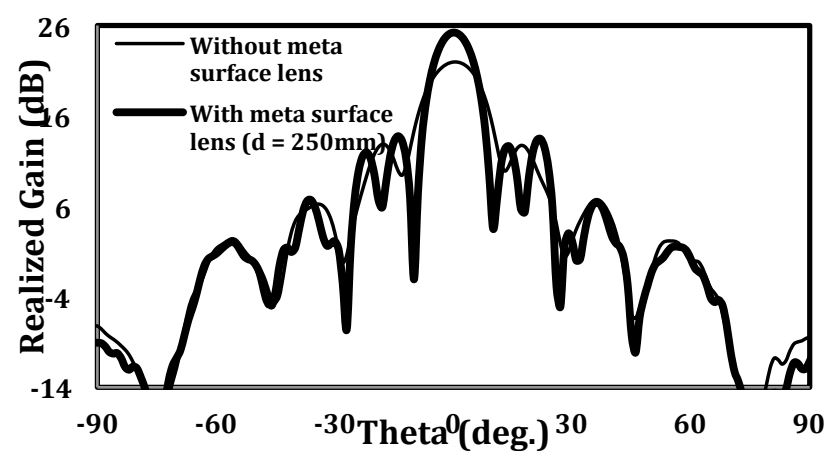

(b)

Figure 5: Simulated far-field radiation patterns in the (a and b) at E -plane of the horn antenna loaded outside with and without meta surface at $9.7 \mathrm{GHz}$ and $10 \mathrm{GHz}$, respectively.

The electric field distributions in E-plane of horn antenna with and without meta surface lens for $9.7 \mathrm{GHz}$ and $10 \mathrm{GHz}$ are shown in Figure 6. From the figure it is observed that with the presence of metasurface lens, antenna is able to focus more field than the without metasurface lens.

The radiation efficiency of meta surface lens loaded horn antenna is numerically calculated from $3 \mathrm{~dB}$ beam width of $\mathrm{E}$ and $\mathrm{H}$ plane at $9.7 \mathrm{GHz}$ and $10 \mathrm{GHz}$ are presented in Table I. From the table it is observed that loading of meta surface lens does not affect the radiation efficiency much but it slightly improved the radiation efficiency for both the frequencies.

Table I: Radiation efficiency of horn antenna with and without meta surface

\begin{tabular}{|l|l|l|}
\hline $\begin{array}{l}\text { Sl. } \\
\text { No. }\end{array}$ & $\begin{array}{l}\text { Frequency } \\
(\mathrm{GHz})\end{array}$ & Radiation Efficiency (\%) \\
\hline 1. & 9.7 & Without meta surface lens=79.46 \\
\cline { 3 - 3 } & & With meta surface lens $=83.3$ \\
\hline 2. & $10 \mathrm{GHz}$ & Without meta surface lens $=74.2$ \\
\cline { 3 - 3 } & & With meta surface lens $=75.3$ \\
\hline
\end{tabular}




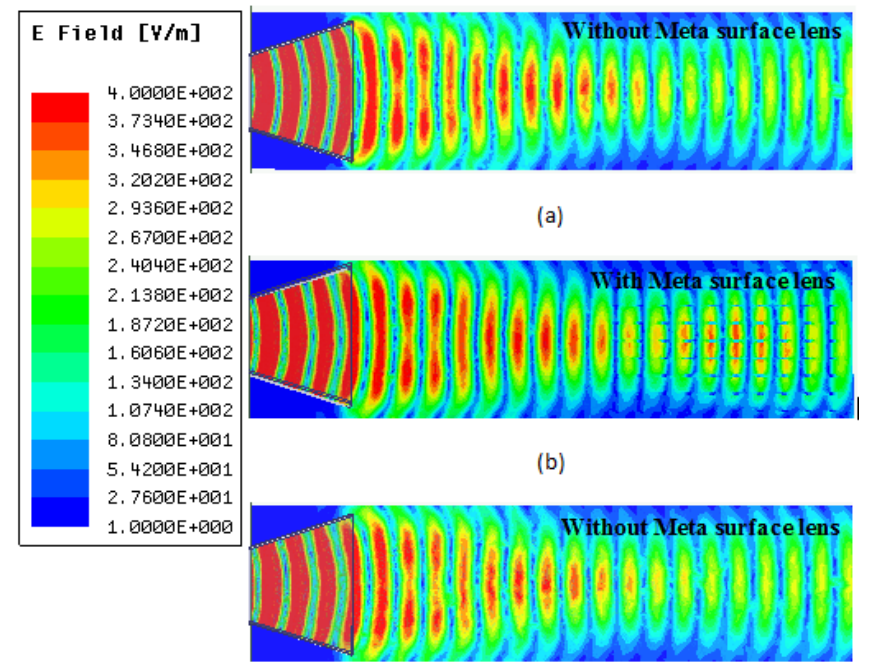

(c)

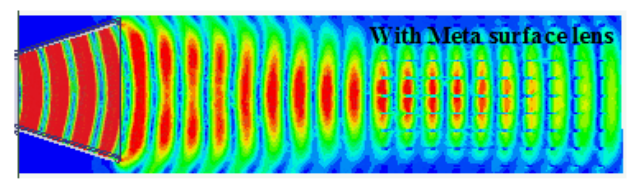

(d)

Figure 6: E-field distribution - (a) and (b) without and with metasurface lens at $9.7 \mathrm{GHz}$, (c) and (d) without and with metasurface lens at $10 \mathrm{GHz}$, respectively.

\section{Experimental Results of Horn Antenna With and Without Meta surface Lens}

The Meta surface lens were fabricated with FR4 strip was placed in front of the horn antenna. Transmitting and receiving horn antenna were X-band and placed 10 feet off the ground on Teflon stands. The test antenna were placed $3 \mathrm{~m}$ away from transmitting antenna and photograph of outdoor setup is show in figure 7. Pattern measurement was measured at $9.7 \mathrm{GHz}$ and $10 \mathrm{GHz}$ by rotating the test antenna in azimuth direction from $-180^{\circ}$ to $+180^{\circ}$. In the beginning power was measured for various gaps (d) between the aperture of horn antenna to (and) the meta surface lens with fixed position of both transmitting and receiving horn antenna. Measured gain at receiver when $-5 \mathrm{dBm}$ power was transmitted from a distance of $3 \mathrm{~m}$ for various gap(d) given in Table II. The E-plane beam pattern at $9.7 \mathrm{GHz}$ and 10 $\mathrm{GHz}$ is shown in figure 8 . The measurement agrees with the simulated results shows that $3 \mathrm{~dB}$ improvement in gain with sharpening of beam at far field with $\pm 0.25 \mathrm{~dB}$ errors. Half power beam width of the horn antenna with meta surface lens were reduced to $8^{\circ}$ from $13^{\circ}$.

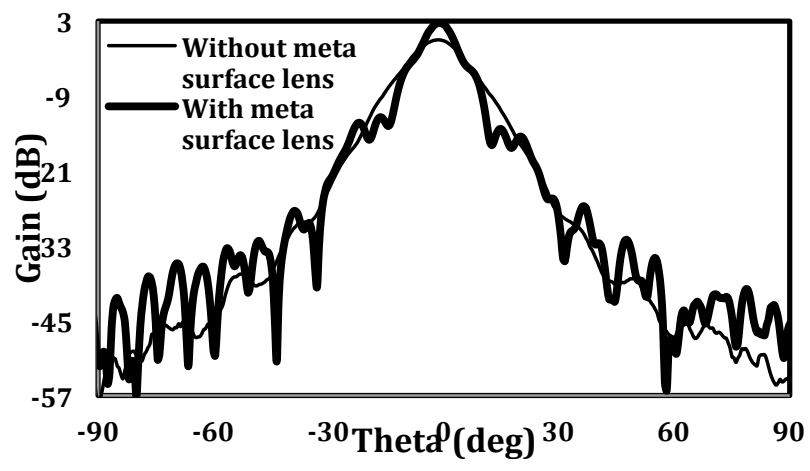

(a)
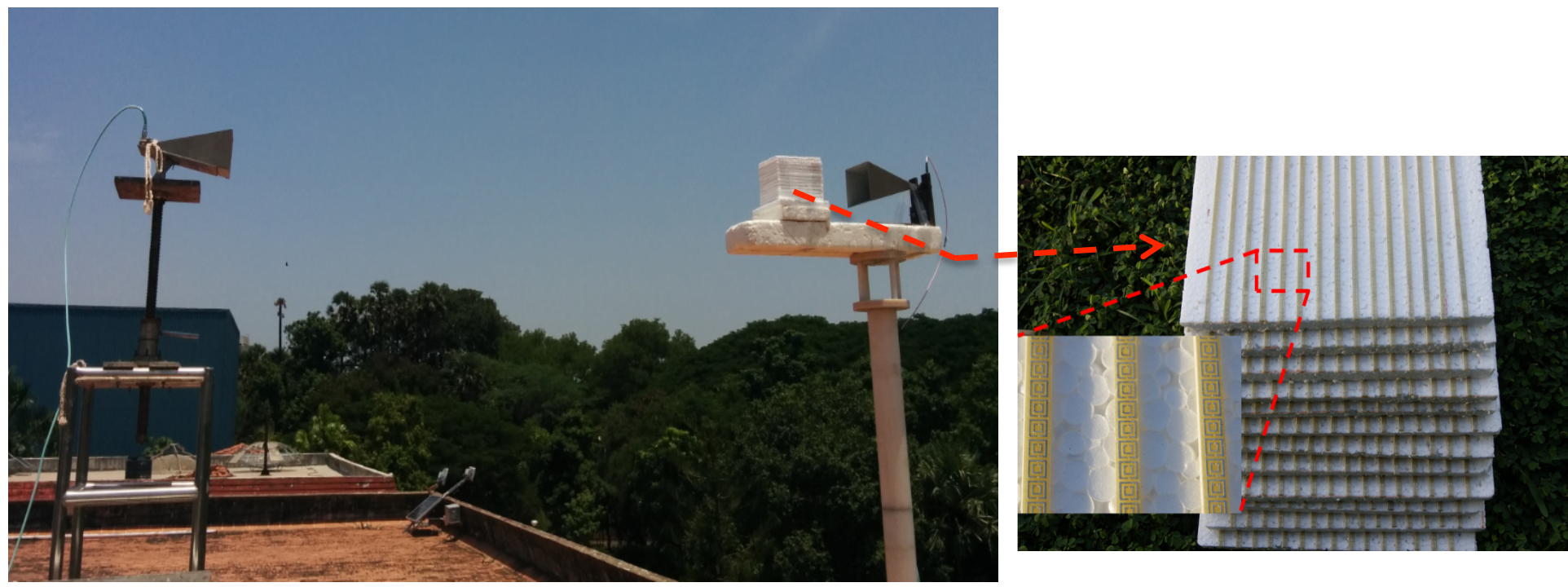

Figure 7: Open area Far field radiation pattern measurement. Top view of fabricated meta surface lens with array of SRR on fr4 strip. Each strip has 49 SRR elements and each layer has 15 strips. Similarly 11 layers are stacked to get 3D lens. 


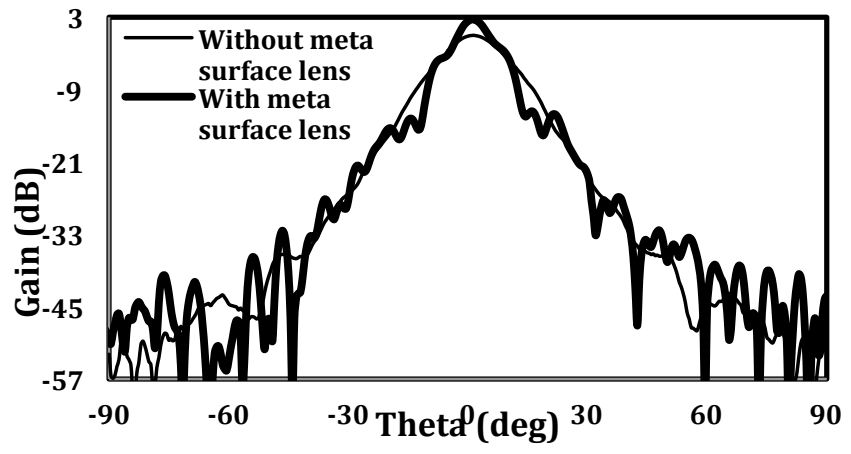

(b)

Figure 8: Measured far-field radiation patterns in the (a and b) at E - plane of the horn antenna loaded outside with and without meta surface at $9.7 \mathrm{GHz}$ and $10 \mathrm{GHz}$ respectively.

Table II: Measured power of horn antenna with and without meta surface

\begin{tabular}{|l|l|l|l|l|l|}
\hline $\begin{array}{l}\text { S1. } \\
\text { No. }\end{array}$ & $\begin{array}{l}\text { Frequency } \\
(\mathrm{GHz})\end{array}$ & $\begin{array}{l}\text { Measured } \\
\text { Gain } \\
\text { Without } \\
\text { Meta } \\
\text { surface } \\
\text { lens (dB) }\end{array}$ & $\begin{array}{l}\text { Gap } \\
(\mathrm{d}) \\
\mathrm{mm}\end{array}$ & $\begin{array}{l}\text { Measured } \\
\text { Gain } \\
\text { With } \\
\text { Meta } \\
\text { surface } \\
\text { lens (dB) }\end{array}$ & $\begin{array}{l}\text { Gain } \\
\text { improved } \\
\text { with Meta } \\
\text { surface } \\
\text { lens(dB) }\end{array}$ \\
\hline & & & 50 & 22.3 & 1 \\
1. & $9.7 \mathrm{GHz}$ & 21.3 & 100 & 22.9 & 1.6 \\
& & & 200 & 23.36 & 2.06 \\
& & 250 & 24 & 2.7 \\
\hline & & & 50 & 24.08 & 2.08 \\
& & & 100 & 24.12 & 2.12 \\
& & & 150 & 24.05 & 2.05 \\
& & & 200 & 24.7 & 2.7 \\
& & & 250 & 24.9 & 2.9 \\
\hline
\end{tabular}

Comparison of proposed work with recently published work is presented in Table III. In the both the cases refractive index is close to zero. But in proposed work gain improvement of $1 \mathrm{~dB}$ is achieved more than the fishnet metamaterial lens antenna.

Table III: Comparison of proposed work with existing work

\begin{tabular}{|l|l|l|l|}
\hline $\begin{array}{l}\text { S1. } \\
\text { No. }\end{array}$ & Title & $\begin{array}{l}\text { Refractive } \\
\text { index(n) }\end{array}$ & $\begin{array}{l}\text { Gain } \\
\text { Improved } \\
(\mathrm{dB})\end{array}$ \\
\hline 1. & $\begin{array}{l}\text { Soret Fishnet Metalens } \\
\text { Antenna (Existing Work) }\end{array}$ & -0.06 & $\sim 2$ \\
\hline 2. & $\begin{array}{l}\text { Gain Enhancement of } \\
\text { Horn Antenna Using Meta } \\
\text { Surface Lens (Proposed } \\
\text { Work) }\end{array}$ & 0.14 & $\sim 3$ \\
\hline
\end{tabular}

\section{Conclusion}

In this paper, meta surface lens hold an array of SRR which was designed and numerically simulated and tested. The obtained results shows that permittivity have negative real parts; this means it becomes resonance at particular range of frequency can able to convert spherical wave into plane wave. Simulated results show that, return loss is weakened slightly after placing a meta surface lens in front of the aperture of horn antenna. E-Plane and H-Plane radiation pattern at main lobe is sharpened obviously and antenna gain is improved approximately $3 \mathrm{~dB}$ with meta surface lens.

\section{References}

[1] A. W. Love, Electromagnetic Horn Antenna. New York: IEEE Press, 1976.

[2] Mei Qing Qi, Wen Xuan Tang, He-Xiu Xu, Hui Feng $\mathrm{Ma}$, and Tie Jun Cui, " Tailoring radiation patterns in broadband with controllable aperture field using meta surfaces," IEEE Trans. Antennas Propag., vol. 61, no. 11, pp. 5792-5798, Nov. 2013.

[3] Mei Qing Qi, Wen Xuan Tang, HuiFeng Ma, Bai Cao Pan, Zui Tao, Yong Zhi Sun and Tie, Jun Cui, "Suppressing side-lobe radiation of horn antenna by loading Meta surface lens," Nat. Scientific Report, pp. 1 -6, Mar. 2015.

[4] Hui Feng Ma and Tie Jun Cui., "Three-dimensional broadband and broad-angle transformation- optics lens," Nat. Commun., pp. 1 - 7, Nov. 2010.

[5] Mei, Z. L., Bai, J., Niu, T. M.andCui, T. J.Ahalf, "A half Maxwell fish-eye lens antenna based on gradient-index meta surfaces," IEEE Trans. Antennas Propag., vol. 60, no. 1, pp. 398-401, Jan. 2012.

[6] Hui Feng Ma, Ben Geng Cai, Teng Xiang Zhang, Yan Yang, Wei Xiang Jiang, and Tie Jun Cui, "Threedimensional gradient-index materials and their applications in microwave lens antennas," IEEE Trans. Antennas Propag., vol. 61, no. 5, pp. 2561-2569, May 2013.

[7] James Baker-Jarvis, and Sung Kim, "The Interaction of Radio-Frequency Fields With Dielectric Materials at Macroscopic to Mesoscopic Scales," Journal of Research of the National Institute of Standards and Tech., Vol.117, pp. 1 -60, Feb. 2012.

[8] V.G. Veselago, "The electrodynamics of substance with simultaneously negative values of epsilon and mu," Sov. Phys. USPEKHI, vol. 10, no.4, pp. 509-514, Feb. 1968.

[9] D.R. Smith, W.J. Padilla, D.C. Vier, S.C. NetmatNasser, and S. Schultz, "Composite medium with simultaneously negative permittivity and permeability," Phys. Rev. Lett., vol. 84, no. 18, pp. 4148-4187, May 2000.

[10] Fan-Yi Meng, Rui-Zhi Liu, Kuang Zhang, Daniel Erni, Qun Wu1, Li Sun, and Le-Wei Li,"Automatic design of 
broadband gradient index meta surface lens for gain enhancement of circularly polarized antennas," Progress In Electromagnetics Research, Vol. 141, pp. 17-32, May 2013.

[11] Guo Qing Luo, Wei Hong, Hong Jun Tang, JiXin Chen, Xiao Xin Yin, Zhen Qi Kuai, and $\mathrm{Ke} \mathrm{Wu}$, "Filtenna Consisting of Horn Antenna and Substrate Integrated Waveguide Cavity FSS," IEEE Transac. Antennas Propag., Vol. 55, No. 1, pp. 92-98, Jan. 2007.

[12] Bakhtiyar Orazbayev, Miguel Beruete, Victor PachecoPena, Gonzalo Crespo, Jorge Teniente and Miguel Navarro-Cia, "Soret Fishnet Metalens Antenna" Nat. Scientific Report, pp. 1-7, Feb. 2015. 